\title{
Werkstattarbeit im Geographieunterricht an Gymnasien - theoretische Überlegungen und Erfahrungen
}

Die Schweiz erlebte in den letzten Jahren einen regelrechten "Werkstattboom». In den meisten Weiterbildungsveranstaltungen über erweiterte Lernformen ist denn auch vom «Werkstattunterricht» die Rede (vgl. auch VOLKART in diesem Heft). Wichtig für die Lehrperson, die mit dieser Methode arbeiten will, sind neben den theoretischen Basisinformationen vor allem Vorschläge zu deren praktischer Umsetzung. In diesem Aufsatz wird der Begriff des Werkstattunterrichts erklärt und auf die Bedeutung dieser Unterrichtsmethode für das heutige Gymnasium eingegangen. Anschließend wird die Durchführung einer Werkstatt paradigmatisch beschrieben. Die dabei aufgetretenen Schwierigkeiten sowie der Gewinn für die beteiligten Mittelschülerinnen und -schüler werden analysiert.

\section{Allgemeine Bemerkungen zum Werkstattunterricht}

\subsection{Was ist Werkstattunterricht?}

Die Bezeichnung «Werkstatt» ruft spontan Gedanken an Arbeiten mit Hammer und Nägeln wach. Eine Lernwerkstatt hat tatsächlich auch etwas mit der Werkstatt eines Handwerkers gemeinsam: In beiden werden nämlich Aufträge ausgeführt, in beiden liegen Arbeitsmaterialien und Werkzeuge geordnet bereit. FREY \& FREY-EILING (1992, Kap. 23, 1 ff.) unterscheiden zwischen dem klassischen Werkstattunterricht, der auf die Reformpädagogik zurückgeht und bei dem die Erstellung eines Werkes im Vordergrund steht, und dem schweizerischen Werkstattunterricht, der einem "vereinfachten Keller-Plan» entspricht. Gemeint ist damit, daß der Stoff in kleine Einheiten aufgeteilt, mit Arbeitsanleitungen, Übungen, Experimenten, Beobachtungsaufträgen usw. versehen und dann in Einzel-, Partner- oder Gruppenarbeit bearbeitet wird. Zeitpunkt und Zeitdauer der Bearbeitung sind frei wählbar. Mittels kleiner Tests können die Teilnehmer ihren Arbeitsstand zwischendurch prüfen (vgl. FREY \& FREYEILING 1992, Kap. 21, 23, Kap. 23, 3). Der schweizerische Werkstattunterricht läßt sich in den Schulen gut realisieren, weshalb auch die meisten bis jetzt publizierten Werkstattvorlagen diesem Ansatz entsprechen.

In der schweizerischen Bildungslandschaft versteht man unter Werkstattunterricht also eine Form des selbständigen und individualisierten Lernens, das in einem vom Lehrer organisierten offenen Arrangement von Lern- situationen und Materialien stattfindet (vgl. GASSER 1992, 167; LANDWEHR 1994, 180; SCHOCH-PERRET 1994, 18). Die Lernwerkstatt leitet an zu Entdeckungs-, Erfahrungs- und Erkundungsprozessen alltäglicher Phänomene, zu experimentellem Lernen, zum Kombinieren, zur Dokumentation des Gelernten, zur Reflexion dieser Lernerfahrung und zur Aufarbeitung der Lernprozesse. Die Inhalte richten sich nach Aktualität, Lebensnähe und der Erlebniswelt der Schülerinnen und Schüler.

Für die Durchführung einer Werkstatt im Schulzimmer werden zu einem bestimmten Thema verschiedene Lernposten eingerichtet, welche von der Lehrperson vorbereitet und strukturiert worden sind. Die Aufgaben können thematisch gebunden oder fächerübergreifend sein. Jeder Lernposten besteht aus einer oder mehreren Lernaufgaben oder Arbeitsaufträgen zu bestimmten Teilthemen sowie den Informationsunterlagen und -materialien, die zur selbständigen Bearbeitung der betreffenden Lernaufgabe gebraucht werden. Die Schülerinnen und Schüler arbeiten nicht alle gleichzeitig an der gleichen Aufgabe, sondern wählen diejenigen Lernposten aus, die sie aufgrund ihres Interesses bearbeiten möchten. Je nach Aufgabe arbeiten sie alleine, zu zweit oder in Gruppen. Die Auswahl und Reihenfolge der Posten kann den Schülern' freigestellt werden; es können aber auch einige Pflichtposten definiert und der Rest als fakultatives Programm (ergänzend und/oder vertiefend) angeboten werden. Die Schüler bestimmen ihren Lernweg also weitgehend eigenverantwortlich und kontrollieren ihren Lernerfolg auch selbst. Jeder Schüler hat einen Werkstattpaß - auch Arbeits- oder Lernpaß genannt (Abb. 1) -, auf dem die Themen der verschiedenen Posten aufgelistet sind. Er dient als Orientierungs-, Planungs- und Kontrollunterlage bei der Bearbeitung des Lernparcours.

Es gibt Werkstattformen, bei denen der Schüler während der Arbeit an jedem Posten ein Lernprotokoll (auch Arbeitsjournal) oder ein Lerntagebuch (auch Lernbericht) führt. Während das Lernprotokoll eher die inhaltliche Seite erfaßt, wird im Lerntagebuch mehr Gewicht auf

${ }^{1}$ Im Text werden die Begriffe Schüler und Lehrer sowohl für männliche als auch für weibliche Personen verwendet.

Sibylle Reinfried, Dr., Geographisches Institut ETH, Winterthurerstraße 190, 8057 Zürich 
Name:

Klasse:

\section{Werkstatt "Wetter und Klima"}

\begin{tabular}{|c|c|c|c|}
\hline Nr. & Thema & \begin{tabular}{|l|}
$\begin{array}{l}\text { Zeit- } \\
\text { bodarf } \\
\text { (Min.): }\end{array}$ \\
\end{tabular} & \begin{tabular}{|l|}
$\begin{array}{l}\text { richiris } \\
\text { becar- } \\
\text { beitet: }\end{array}$ \\
\end{tabular} \\
\hline 1 & Geschichte der Wetterbeobachtung & $10^{\prime}$ & \\
\hline 2 & Kondensation von Wasserdampf & $15^{\prime}$ & \\
\hline 3 & Bildung von Wolken & $12^{\prime}$ & \\
\hline 4 & Konvektion & $15^{\prime}$ & \\
\hline 5 & Wolkentypen eines dynamischen Tiefs & $10^{\circ}$ & \\
\hline $6 \mathrm{P}$ & Inversion & $15^{\circ}$ & \\
\hline 7 & Blitz und Donner & $15^{\circ}$ & \\
\hline 8 & Hagel und Hagelschäden & $15^{\circ}$ & \\
\hline 9 & Satelliten beobachten das Wetter & 12 & \\
\hline 10 & Wetterlage Europas & $10^{\circ}$ & \\
\hline $11 \mathrm{P}$ & Kälteempfinden & $20^{\circ}$ & \\
\hline 12 & Wetterfühligkeit bei Föhn & $10^{\circ}$ & \\
\hline 13 & Bauemregeln & $10^{\circ}$ & \\
\hline 14 & Sonneneinstrahlung & $15^{\circ}$ & \\
\hline $15 \mathrm{P}$ & FCKW - Killer der Ozonschicht & $10^{\circ}$ & \\
\hline 16 & Abbau der Ozonschicht & $10^{\circ}$ & \\
\hline 17 & Klimaänderung in der Vergangenheit & $10^{\circ}$ & \\
\hline 18 & Klimaänderung in der Zukunft & 15 & \\
\hline
\end{tabular}

Die Pnichtposten sind fett gedruckt.

Die normal gedruckten Posten sind freiwillig. Bearbeite mindestens fün Posten nach eigener $W$ ahl.

$P$ bedeutet Parnerarbeit, die übrigen Posten werden einzeln bearbeitet.

Abb.1 Beispiel für einen Werkstattpaß.

den Lernprozeß und das Sozialverhalten gelegt. Im Sinne eines Lerndialoges gibt der Lehrer zum Lerntagebuch eine schriftliche oder mündliche Rückmeldung: Er kommentiert Entwicklungsfortschritte, weist auf hilfreiche Formen und Techniken des Lernens hin oder bietet Hilfen für Verbesserungen an (SCHOCH-PERRET 1994, 18; LANDOLT 1994, 100). Werkstattlernen soll somit sowohl Lernerlebnisse ermöglichen und Lust am Lernen bewirken als auch zu überprüfbaren und bewertbaren Lernergebnissen führen (GASSER 1992, 167; 1995, 251 ff.).

Individualisiertes Lernen darf nicht mit Einzelunterricht gleichgesetzt werden. Das Arbeiten mit einem Partner oder in Gruppen zeigt, daß dem sozialen Lernen und der Gemeinschaftsbildung auch bei dieser Unterrichtsmethode höchste Bedeutung zukommen (REICHEN 1991, 63). Kommunikationsfähigkeit, Entscheidungsfähigkeit, Ausdauer und Toleranz der Schüler werden trainiert. Erfahrungen zeigen, daß im Werkstattunterricht die Hilfsbereitschaft unter den Schülern, der Wille und Wunsch zur Zusammenarbeit größer sind als im herkömmlichen Frontalunterricht.

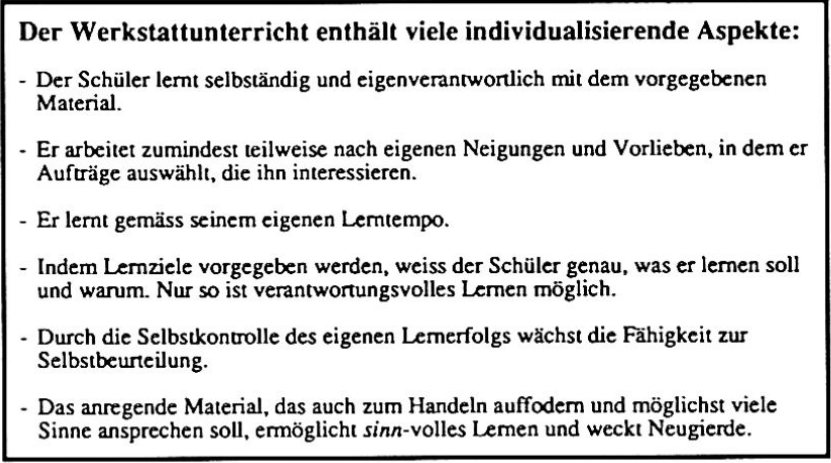

Abb.2 Individualisierende Aspekte des Werkstattunterrichts.

\subsection{Neue Rolle der Lehrperson}

Werkstattunterricht verlangt Mut - sowohl von Schülern als auch von Lehrpersonen. Die Schüler müssen sich auf eine neue Unterrichtsmethode einlassen, die andere Fähigkeiten und Fertigkeiten als der Frontalunterricht von ihnen verlangt. Der Lehrer wiederum wird mit einer neuen Rolle konfrontiert. Er ist nicht mehr der, der «belehrt», sondern wird zum Lernbeobachter, Zuhörer und Lernberater, der sein Sachwissen nur mehr dosiert und punktuell, dafür aber auf hohem Niveau einsetzen kann. Öffnung des Unterrichts bedeutet somit nicht nur, daß die Lehrperson methodische Neuerungen in ihren Unterricht einführt, sondern beinhaltet auch eine starke Konfrontation mit dem eigenen Rollenverständnis als Lehrkraft. Öffnung des Unterrichts ruft daher bei vielen Lehrern Ängste und Unsicherheiten hervor: Angst vor Kontrollverlust und Überforderung durch zu große Vielfalt und zu komplexe Lernsituationen, aber auch Angst, das Pensum des Lehrplans nicht zu schaffen.

Nichtlehrerzentrierte Lernformen lassen den Schülern auch mehr Raum für Unterrichtsstörungen. Da diesen meist persönliche Lernschwierigkeiten oder gruppendynamische Probleme zugrunde liegen, fordert dieser Unterricht vom Lehrer eine hohe Präsenz, die vor allem für denjenigen, der bisher frontal unterrichtet hat, neu ist. Auch wenn die neue Rolle von der Lehrkraft einiges an Flexibilität, Stabilität sowie die Fähigkeit, den Lernproblemen und den schulischen sowie persönlichen Fragen der Schüler mit Einfühlungsvermögen zu begegnen, abverlangt, sollte dies den Lehrer nicht vom Werkstattunterricht als Teil eines interessanten, abwechslungsreichen, methodisch breit abgestützten Unterrichts abhalten. Den Lehrern, die feststellen, daß sie in ihrer Ausbildung nicht auf die neuen Anforderungen vorbereitet wurden, steht heute ein breites Fortbildungsangebot (vgl. Kurse zur Unterrichtspraxis in den Kursprogrammen der WBZ Luzern) zur Verfügung. 


\subsection{Zum Entstehungshintergrund der Lernwerkstätten}

Lernwerkstätten sind als eine Gegenreaktion auf die vor allem für die Naturwissenschaften formulierten "geschlossenen Curricula» aus den 70er Jahren zu verstehen. Diese waren durch einen stark vorstrukturierten Lernprozeß geprägt, der sich vor allem in der sehr genauen Ausformulierung von Lernzielen, der sogenannten «Operationalisierung von Lernzielen», äußerte. Die geschlossenen Curricula gewährten entdeckendem, selbstbestimmtem und erfahrungsorientiertem Lernen wenig Spielraum. Die im Gegenzug entwickelten "offenen Curricula» lösten die starke Vorstrukturierung von Lernprozessen wieder mehr zugunsten einer Präsentation von offenen Lernangeboten auf (PALLASCH \& REIMERS 1990, 21). Der kopflastige, leistungsorientierte Unterricht wird zunehmend durch ein Lernen mit "Hand" und "Herz» ergänzt. Das Schlagwort heisst Individualisierung. Gemeint ist damit ein ganzheitlicher Unterricht, der nicht nur Denken, Planen und Handeln in sich vereint, sondern auch Wahlfreiheit und individuelles Lernen zuläßt. Die Forderung nach vermehrter Individualisierung im Unterricht ist auch ein Ergebnis der erziehungswissenschaftlichen Diskussion der 70er Jahre, die sich schwerpunktmäßig mit Fragen der Chancengleichheit im Bildungsprozeß befaßt hat. Verlangt wird, daß im Unterricht Schüler aller Begabungen gefördert werden (PALLASCH \& REIMERS 1990, 98 f.). Dazu bedarf es jedoch anderer, partizipativer Unterrichtsmethoden, die nicht nur auf reiner Wissensvermittlung beruhen. Das Werkstattlernen entspricht einer solchen Unterrichtsmetho$\mathrm{de}$, da es erfahrungsorientierte, entdeckende, ganzheitliche und selbstbestimmte Lernprozesse zuläßt (Abb. 2). Neuerdings wird der Ruf nach schüler- und erfahrungsorientierten Lernformen auch von der Kognitionspsychologie gestützt, welche ausgehend von einem veränderten Menschenbild und einem dadurch veränderten Verständnis von Lernprozessen, den Menschen als ein früh-autonomes, reflexiv denkendes, selbstverantwortlich handelndes, nach Selbständigkeit strebendes Subjekt versteht, das sich eigene Lernziele setzt und eigene Handlungskonzepte entwickelt (EDELMANN 1993, 304 ff.; PALLASCH \& REIMERS 1990, 46). Neue Erkenntnisse aus der Unterrichtsforschung bestätigen diese Analysen (KELLER 1995).

\subsection{Chancengleichheit}

Wahrt der Werkstattunterricht die Chancengleichheit? Schafft der Werkstattunterricht nicht Ungerechtigkeiten, da nicht alle Schüler genau den gleichen Stoff lernen?

Diese Fragen beruhen auf der Annahme, daß Chancengleichheit nur dann möglich ist, wenn alle Schüler die genau gleichen Lernmöglichkeiten haben. Dabei wird nicht berücksichtigt, daß verschiedene Wege zum Verständnis eines (natur)wissenschaftlichen Prinzips führen. Da jeder Mensch unterschiedliche Lernvoraussetzungen mitbringt, kann gerade ein einheitliches Lernangebot bestehende Unterschiede verfestigen. Wer echte Chancen- gleichheit anstrebt, muß den Schülern auch individualisierte Bildungsangebote machen (GASSER 1992, 168).

Daraus soll nun aber nicht der Anspruch abgeleitet werden, daß der Unterricht vermehrt werkstattartig durchzuführen ist, denn auch zuviel Werkstattunterricht zeitigt bei den Schülern gewisse Ermüdungserscheinungen. Häufige Werkstätten übersteigen aufgrund ihrer aufwendigen zeitlichen und organisatorischen Planung auch die Möglichkeiten vieler Lehrkräfte und Schulen. Wo Werkstattunterricht oder eine andere erweiterte Unterrichtsform angebracht ist und wo nicht, hängt letztlich sowohl vom Thema, das im Unterricht behandelt werden soll, als auch von den Zielen, die mit diesem Unterricht angestrebt werden, ab. Jede Unterrichtsmethode hat bekanntermaßen neben ihren Vorteilen auch Nachteile.

\section{Werkstattunterricht am Gymnasium}

\subsection{Wozu Werkstattunterricht am Gymnasium?}

Die Forderung nach einer neuen Lernkultur erstreckt sich heute auf den gesamten Bildungsbereich, also auch auf Gymnasien und Hochschulen. Als Hauptargument wird oft die Tatsache genannt, daß heute an Auszubildende und Berufsleute erhöhte Anforderungen bezüglich Aufgabenwahrnehmung, Eigeninitiative, Mit- und Selbstverantwortung gestellt werden, während reine Ausführungstätigkeiten zunehmend an Bedeutung verlieren (WEBER 1991, 13 f.). Neben Fachwissen, das immer mehr zunimmt, aber auch immer schneller veraltet, ist heute vermehrt auch Zusammenhangswissen, Planungs-, Steuerungs- und Analysewissen gefragt, das durch reine Lehrveranstaltungen in der Art des Frontalunterrichts alleine nicht vermittelt werden kann (LANDOLT 1994, 85). Verlangt werden zeitgemäßere Unterrichtsformen, also ein Wechsel vom bisherigen Lehrsystem zu einem ganzheitlichen, schüler- und erfahrungsorientierten Unterricht.

Zum Ausdruck kommen diese Überlegungen auch im neuen Reglement zur Anerkennung von gymnasialen Maturitätsausweisen, kurz MAR genannt (SCHWEIZERISCHER BUNDESRAT \& EDK 1995, 3), wo unter Art. 5.2. folgendes Bildungsziel formuliert ist: "Maturandinnen und Maturanden sind fähig, sich den Zugang zu neuem Wissen zu erschließen, ihre Neugier, ihre Vorstellungskraft und ihre Kommunikationsfähigkeit zu entfalten sowie allein oder in Gruppen zu arbeiten. Sie sind nicht nur gewohnt, logisch zu denken und zu abstrahieren, sondern haben auch Übung im intuitiven, analogen und vernetzten Denken..."

Welche Folgerungen ergeben sich hieraus für das Gymnasium? Auch die Mittelschulen müssen den Anforderungen an eine zeitgemäße Bildungsinstitution genügen und das Wissen um den Wandel in der Berufswelt und die Erkenntnisse aus der Unterrichtsforschung mit einbeziehen. Eine Schule, die dem Rechnung trägt, wandelt sich zu einem kooperativen Lernbegegnungs- und -arbeitsort, der die Persönlichkeit und die Ziele des Lernenden ernst 
nimmt und Schülern so ermöglicht, selbstorganisiertes und selbstverantwortliches Lernen zu lernen. Dieses individualisierte Lernen wird, wie der bisherige Unterricht auch, auf dem Hintergrund von curricularen und schulorganisatorischen Vorgaben geplant. Da zurzeit an allen Gymnasien neue Lehrpläne erarbeitet werden, bietet sich die einmalige Gelegenheit, die für individualisierte Unterrichtsformen notwendigen Rahmenbedingungen zu schaffen.

\subsection{Hinweise zur Durchführung einer Werkstatt}

Eine Werkstatt kann eingesetzt werden zu Beginn einer Lerneinheit, als sogenannte Erfahrungswerkstatt, oder am Ende einer Lektionenreihe als Übungswerkstatt (GASSER 1992, 143; ZÜRCHER 1987, 15 f.). Die Dauer einer Werkstatt richtet sich nach der Anzahl und Bearbeitungszeit (10-30 Minuten) der Posten. Man kann schon mit wenigen Posten eine Werkstatt durchführen (GASSER 1992, 167), dann aber in mehrfacher Postenausführung. Damit ein vielfältiges Lernangebot entsteht, empfiehlt sich jedoch, daß die Anzahl der Aufträge etwa ein Drittel höher ist als die Anzahl der Schüler (FREY \& FREY-EILING $1995,10)$. Ideal ist es, wenn man einen Raum zur Verfügung hat, in dem man die Werkstatt stehenlassen kann, so daß man längere Zeit (z. B. einige Tage oder Wochen) daran arbeiten kann. Wenn dies nicht möglich ist, empfiehlt sich mindestens ein Block von 3-4 Stunden.

Der Praktiker, der diese Unterrichtsform durchführen möchte, fragt zuerst nach fertigen, einsetzbaren Vorlagen. Sucht man als Geographielehrer solche im Buchhandel, so findet man lediglich Lehrmittel für die Primarschule (BERIGER 1992; FREI \& HOFFMANN 1992). Für den Geographieunterricht auf der Mittelschulstufe gibt es bis jetzt keine erprobten Werkstattunterlagen, die auch den methodischen Anforderungen genügen. Einige Verlage bieten aber interessantes Unterrichtsmaterial an, welches sich durchaus mit etwas Phantasie zu Werkstattposten umarbeiten läßt (z. B. SLABY 1993; STASCHEIT \& KNEIP 1991).

Was muß man nun beachten, wenn man die Posten selber konzipieren will? Zuerst braucht man eine gute Materialsammlung und interessante Aufgaben. Der Lernparcours kann anschließend nach dem gedächtnispsychologischen wie auch nach dem erkenntnispsychologischen Lernverständnis aufgebaut werden (LANDWEHR 1994, $180 \mathrm{ff}$.). Gedächtnispsychologisches Lernverständnis heißt: Bei der Bearbeitung der Posten wird bekanntes Wissen reproduziert, vertieft, geübt und gefestigt. Wenn man eine Lernwerkstatt oder zumindest einige Posten nach den Grundsätzen der erkenntnispsychologischen Wissensvermittlung ausrichten will, dann werden an den Posten neue Erkenntnisse gewonnen. Damit dieses Ziel erreicht werden kann, muß ein Posten wie folgt aufgebaut sein (Abb. 3):

a) Mit dem Posten wird eine Aufgabenstellung bearbeitet, die dem Schüler aktives Entdecken ermöglicht. Die Lernaufgaben des Postens werden so konzipiert, daß ein Erkenntnisprozeß, der zum beabsichtigten Wissen führt, für den Schüler nachvollziehbar wird (LANDWEHR 1994, 43).

b) Der Posten enthält eine Hinführung zur Problemstellung, in der der Schüler eine Vorstellung von der zu bearbeitenden Aufgabe erhält.

c) Die mit dem Posten anvisierten Lernziele sind angegeben, damit der Schüler weiß, was ihm die Beschäftigung mit der Frage bringt.

d) Die nun folgende Arbeitsanleitung soll dazu einladen, Recherchen anzustellen, eigene Erfahrungen zu sammeln und neue Erkenntnisse zu gewinnen. Der Posten muß alle dazu benötigten Hilfestellungen enthalten, damit die Hypothesenbildung einigermaßen fruchtbar ausfällt. Der Schüler muß wissen, wie er das beim Posten vorhandene Material verwenden muß.

e) Die Postenbeschreibung enthält Angaben über die benötigte Arbeitszeit, die zu wählende Sozialform (Einzel-, Partner-, Gruppenarbeit) und eine Liste des zur Verfügung stehenden Materials oder anderer Lernhilfen (z. B. Bücher, Lexika).

f) Nach getaner Arbeit will der Schüler seine Lösung überprüfen. Erkenntnisorientierte Arbeitsaufträge führen selten zu einfachen Richtig-Falsch-Antworten. Folglich müssen komplexere Evaluationsformen überlegt werden: Die Lernkontrolle kann beispielsweise durch den Lernpartner, die Lehrperson, durch Vergleich mit einem Fachtext oder mit Lösungsblättern, in einer Plenumsdiskussion, durch Vorträge, durch einen Test oder durch eine Prüfung erfolgen.

Grundsätzlich gilt für jeden Posten, ganz gleich ob er nach gedächtnispsychologischem oder erkenntnispsychologischem Lernverständnis konzipiert ist, das folgende:

- Der Schüler sollte möglichst viele Sinne bei der Lösung der Aufgaben einsetzen können, also nicht nur schreiben und lesen, sondern auch zeichnen, experimentieren, beobachten, spielen, diskutieren, herausfinden, erleben, erfahren, ordnen usw. Der Arbeitsauftrag kann auch aus dem Klassenzimmer hinausführen (z. B. Wolkenbeobachtungen).

- Wichtig ist, daß den Schülern die Lernziele der einzelnen Posten transparent gemacht werden.

- Die Arbeitsaufträge müssen klar und verständlich formuliert sein.

- Die Aufgaben sollen unabhängig voneinander bearbeitet werden können.

- Der Lehrer muß Versuche vorher ausprobieren, Aufgaben vorher lösen.

- Der Lehrer entwirft einen Werkstattpaß, in den der Schüler einträgt, welche Posten er richtig bearbeitet hat.

- Die Schüler müssen ihre Lösungen selber kontrollieren bzw. korrigieren können.

Um den Sinn und die Bedeutung des Werkstattlernens zu verstehen und einen reibungslosen Ablauf des Unterrichts zu garantieren, werden die Schüler vor Beginn der Werkstatt in die Methodik dieser Unterrichtsform eingeführt (vgl. GASSER 1995, 253 ff.). Dabei wird ein Minimum an Verhaltensregeln festgelegt. Dann organisieren 


\section{Konvektion}

\begin{tabular}{|c|c|}
\hline Lernziele: & $\begin{array}{l}\text { - Durch Beobachten eines einfachen Experiments verstehst } \\
\text { Du das Verhalten von warmer Luft. }\end{array}$ \\
\hline Zeit: & - 15 Minuten. \\
\hline Einführung: & $\begin{array}{l}\text { Vertikale Luftbewegungen sind die Ursache einer } \\
\text { bestimmten Form von Niederschlagsereignissen, wie sie } \\
\text { bei uns vor allem in den Sommermonaten vorkommen. Im } \\
\text { folgenden Experiment kannst Du vertikale Bewegungen im } \\
\text { Wasser beobachten, welches hier stellvertretend für Luft } \\
\text { verwendet wird. }\end{array}$ \\
\hline
\end{tabular}

Arbeitsanleitung: - Einzelarbeit

1. Fülle das Becherglas zu Beginn des Versuchs mit kaltem Wasser.

2. Nimm den Erlenmeyerkolben und fülle ihn mit warmem Wasser auf.

3. Färbe nun das warme Wasser mit einer Wasserfarbe ein, bis das Wasser eine satte Farbe hat.

4. Schliesse danach den Erlenmeyerkolben mit dem Stöpsel.

5. Stelle den Kolben in das Becherglas und löse dem Stöpsel vorsichtig.

6. Halte den Stöpsel mit zwei Fingern unten und warte 10 Sekunden bis sich das Wasser im Becherglas beruhigt hat.

7. Ziehe nun den Stöpsel aus dem Erlenmeyerkolben (und aus dem Becherglas).

8. Beobachte, was geschieht.

- Deine Aufgabe ist es, den Versuch durchzuführen und dabei Deine Beobachtungen aufzuschreiben.

- Leite aus Deinen Beobachtungen eine allgemeingültige Gesetzmässigkeit ab.

- Welches Wetterereignis lässt sich am ehesten mit Deinen Beobachtungen vergleichen?

Material: $\quad$ Hohes Becherglas

- Kleiner Erlenmeyerkolben (50 ml) mit Kork- oder GummiStöpsel mit Schnürchen

- Wasserfarbe

- warmes und kaltes Wasser 
die Schüler ihre Arbeit selbst. Jeder Schüler überlegt sich, auf welchem Weg er die gestellten Aufgaben lösen will. Nach Abschluß der Werkstatt erfolgt eine Lernkontrolle.

Und wie verhält es sich mit dem Arbeitsaufwand für die Lehrperson? Der Arbeitsaufwand ist tatsächlich groß und erfordert einiges an didaktischer Phantasie. Wenn die Werkstatt aber einmal konzipiert ist, kann man sie immer wieder verwenden. Die Lehrperson erhält somit einen Teil der aufgewendeten Zeit wieder zurück. Da sich Werkstattunterricht für fächerübergreifendes Lernen sehr eignet, bietet es sich an, die Vorarbeiten im Team durchzuführen.

\section{Erfahrungsbericht aus der Praxis}

\subsection{Die Werkstatt "Wetter und Klima»}

Im folgenden werden die Durchführung und die Erfahrungen mit der Werkstatt «Wetter und Klima» im Geographieunterricht beschrieben.

Die Werkstatt «Wetter und Klima» wurde von Geographielehrerstudenten im Didaktikkurs «Fachspezifische Unterrichtsmethoden der Geographie», den ich in meiner Funktion als Fachdidaktikerin jeweils im Sommersemester an der ETH Zürich anbiete, entworfen. Die Posten sollten so konzipiert werden, daß bekanntes Wissen abgerufen und auf Alltagssituationen übertragen wird. Die praktischen Anwendungsbeispiele sollten es den 15bis 16jährigen Schülern ermöglichen, dieses eher abstrakte Thema, das große Anforderungen an das Vorstellungsvermögen von Jugendlichen in diesem Alter stellt, besser zu verstehen. Bis jetzt habe ich den Parcours zweimal in einer 3. Gymnasialklasse (9. Schuljahr) des Freien Gymnasiums in Zürich getestet. Er wurde auch dem Kollegium des Freien Gymnasiums und den GeographiePraktikumslehrern der Universität und der ETH Zürich an einem Weiterbildungstag vorgestellt. Aus der Diskussion ergaben sich zahlreiche Verbesserungsvorschläge. und Anregungen, die anschließend umgesetzt wurden. Der Lernparcours wurde als Übungswerkstatt konzipiert, die die Unterrichtsreihe "Wetterkunde» abschließt. Darauf folgte eine Prüfung über den gesamten Stoff. Der Lernparcours, der aus 18 Posten besteht, wurde im Singsaal der Schule aufgebaut. Für die Durchführung wurde ein Block von drei Schulstunden (140 Minuten) an einem Nachmittag geplant. Der Block wurde möglich dank Stundenabtausch mit anderen Fachlehrern. Auf jedem Tisch wurde ein Posten mit Informations- und Arbeitsblättern in genügender Anzahl und dem benötigten Arbeitsmaterial eingerichtet.

Die Werkstatt ist wie folgt aufgebaut: Die meisten Posten sind einzeln zu bearbeiten, an drei Posten ist aber auch Partnerarbeit vorgesehen, die auf dem Werkstattpaß mit $\mathrm{P}$ gekennzeichnet sind (Abb. 1). Es gibt Pflichtposten und freiwillige Posten. Die Pflichtposten müssen alle, von den freiwilligen Posten sollten mindestens 5 bearbeitet werden. Damit die Schüler die Posten sofort finden, werden die Tische deutlich mit den Postennummern bezeichnet. Die Pflichtposten erhalten eine farbige Numerierung, die freiwilligen Posten ein weißes Nummernkärtchen. Damit für die Schüler keine Wartezeiten entstehen, werden einige Posten auch doppelt und dreifach geführt. Nach Bearbeitung eines Postens sollen die Schüler ihr Lösungsblatt mit den richtigen Lösungen vergleichen und nötigenfalls ihre eigenen Antworten ergänzen. Bei einer falschen Lösung sollen sie mit mir gemeinsam die Ursache der Fehler herausfinden. Erst darauf halten sie mein Testat auf dem Werkstattpaß, so daß sie weiterfahren können.

\subsection{Eigene Erfahrungen...}

Vor Werkstattbeginn erklärte ich den Klassen kurz Ziele und Ablauf des Werkstattunterrichts. Anschließend verteilte ich die Werkstattpässe. Dann folgten einige wenige disziplinarische Vorgaben, so z. B., daß das Postenmaterial an seinem Platz bleiben muß und daß Störungen zu vermeiden sind.

Beim Eintreten in den Singsaal stürzten sich die 20 Schüler und Schülerinnen sofort auf die Pflichtposten, von denen es aber nur 8 (einige davon in doppelter Ausführung) gab (Abb. 4 und 5). Da diese schnell besetzt waren, mußten sie sich unter dem Angebot der freiwilligen Posten umsehen und sich für eines jener Themen entscheiden. An den Posten, an denen kleine Experimente vorgesehen sind, benötigten einige Schüler trotz der eindeutigen Versuchsanleitungen zusätzliche Hilfestellungen. Ursache dafür war die Unsicherheit in der Handhabung der Geräte, die mangelnde Übung in der Durchführung von Versuchen, aber auch die wenig geschulte Beobachtungsfähigkeit.

Unerwarteterweise schlossen sich die Schülerinnen und Schüler zu Beginn des Unterrichts spontan zu Zweierund Dreiergruppen zusammen, obwohl bei den meisten Posten Einzelarbeit vorgesehen war. Diese Gruppenbildung sollte helfen, die Unsicherheiten im Umgang mit der ungewohnten Unterrichtsform zu überwinden. Im Laufe des Nachmittags lösten sich die Gruppen teilweise wieder auf, da die Schüler jetzt offenbar mit der Situation zurechtkamen.

Auffallend war, daß die zu lösenden Aufgaben in den Gruppen so gründlich und engagiert diskutiert wurden, wie ich es bisher im Unterricht noch kaum hatte feststellen können. Dies hat zum Teil damit zu tun, daß die Schüler während der Werkstatt selber gemerkt haben, was sie können. Das hat ihnen Selbstvertrauen gegeben und ihre Bereitschaft gestärkt, auch auf andere einzugehen.

Durch das selbständige Arbeiten im eigenen Lerntempo wurde den Schülern klar, ob sie den Stoff verstanden haben und wo noch Lücken vorhanden sind. Die trockene Theorie verband sich langsam mit konkreten Vorstellungen. Sogenannte "Aha»-Erlebnisse wurden vermittelt und neue Fragen aufgeworfen. Den Schülern, die selbst oder zu zweit vernünftige Lösungen erarbeitet hatten, war Freude und Stolz über die gelungene Leistung anzumerken. 


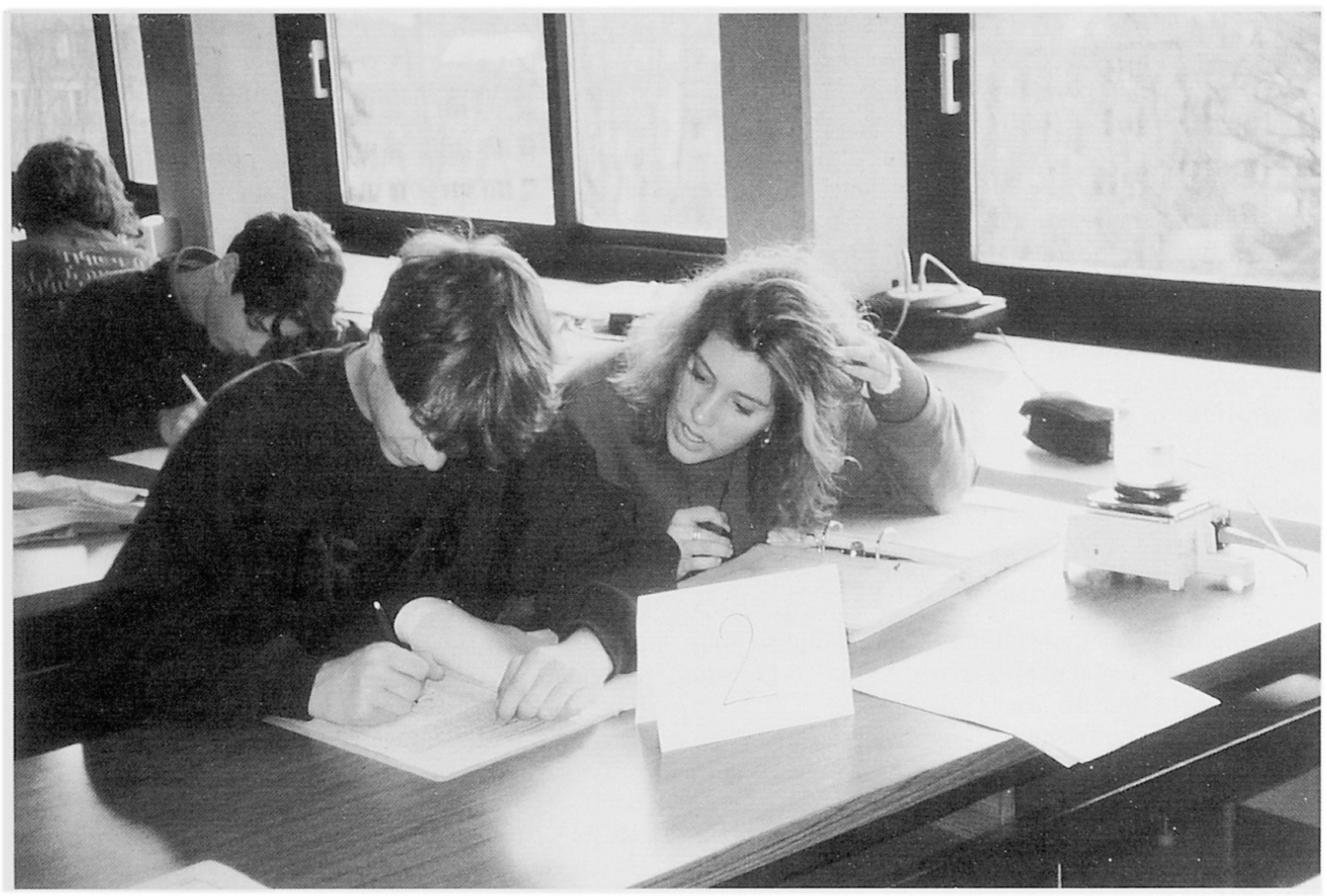

Abb. 4 Der Posten Nr. 2, "Kondensation von Wasserdampf”, wird hier in Partnerarbeit bearbeitet.

Vor allem die Mädchen nutzten die Möglichkeit, ihre Fehler mit mir zu diskutieren. Sie benutzten diese Gespräche auch dazu, sich gewisse Sachverhalte nochmals erklären zu lassen. Die Knaben machten im allgemeinen seltener Gebrauch von dem Diskussionsangebot. Ein Schüler schrieb nur die Lösungsblätter ab, ohne die Posten zu bearbeiten. Zwei andere versuchten zwar die Aufgaben zu lösen, gaben aber immer wieder sehr schnell auf, wenn sie nicht sofort dahinterkamen, was die Lösung des Problems sein könnte, und bedienten sich dann der Lösungsblätter.

Wie sind diese Verhaltensweisen zu erklären? Für alle erweiterten Lernformen gilt, daß Schüler, die bis jetzt überwiegend frontal unterrichtet wurden, sich zuerst an eigenverantwortliches Lernen gewöhnen müssen. Dies trifft auch für den Werkstattunterricht zu. Die drei letztgenannten Schüler waren vom Werkstattunterricht offenbar überfordert. Sie waren nicht fähig, für ihr Lernen Verantwortung zu übernehmen und sich die benötigte Unterstützung zu holen. Hier zeigt sich, wie wichtig es ist, daß sich die Schüler ihres eigenen Lernverhaltens bewußt werden. Ein Lerntagebuch, auf das ich bei dieser Werkstatt verzichtet habe, hätte hier sicher gute Dienste geleistet.
Die vorgesehene Bearbeitungszeit von 140 Minuten hat sich angesichts der Menge der Posten in beiden Klassen als zu kurz erwiesen. In der zur Verfügung stehenden Zeit bewältigten die Knaben und Mädchen 8-10 der vorgegebenen 13 Posten. In einem nächsten Durchgang wäre somit die Bearbeitungszeit um eine weitere Unterrichtsstunde zu verlängern oder aber die Postenzahl zu reduzieren.

Und wie war es mit der Disziplin? Wo Jugendliche sind, gibt es Unterrichtsstörungen, und es wird auch einmal Unsinn gemacht. Mit etwas Humor konnten diese Situationen aber gemeistert werden. Auch der Lärmpegel, der durch die Diskussionen in den Arbeitsgruppen entstand, war höher als im streng geführten Frontalunterricht. Er könnte wahrscheinlich durch eine geschicktere Anordnung der Einzel- und Partnerarbeitsposten, z. B. in zwei Fachzimmern, vermindert werden.

\section{3 ... und die Meinungen der Schülerinnen und Schüler}

Nach Abschluß der Werkstatt wurden die Klassen nach ihrer Meinung über die einzelnen Posten und zur Gesamtwerkstatt befragt. Am besten angekommen waren die Posten, bei denen ein Experiment durchgeführt wer- 


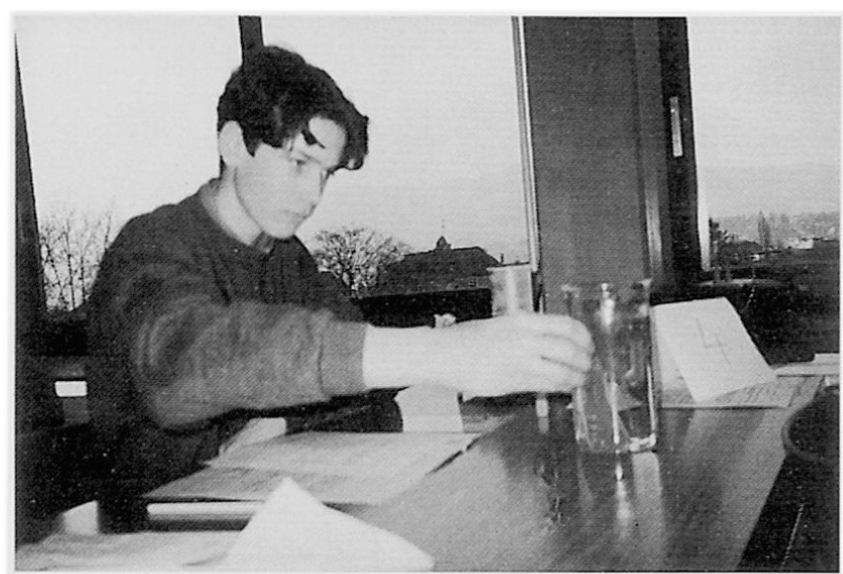

Abb. 5 Posten Nr. 4, "Konvektion». Schüler bei der Durchführung eines Experiments.

den konnte. Posten, bei denen viel gelesen oder geschrieben werden mußte, waren hingegen weniger beliebt. Gewünscht wurde auch mehr Gruppenarbeit und mehr Zeit zur Bearbeitung der Lernaufgaben. Auch wenn man über die "lockere Atmosphäre und den Spaß an den Posten» sehr froh war, hat die Unruhe doch gestört. Einige Lernaufgaben wurden auch als zu schwierig empfunden.

Trotz dieser Kritik wurde die Werkstatt «Wetter und Klima" als eine sehr willkommene Abwechsung zum Normalunterricht geschätzt. Gefallen hat, daß man das Gelernte "so richtig erleben» konnte, etwas «beobachten konnte und sich jetzt aufgrund der eigenen Beobachtung eine bessere Vorstellung davon machen kann", daß man «selber experimentieren» konnte, den Stoff «endlich kapiert hat», die Freiheit hatte, "die Reihenfolge der Posten selber zu bestimmen», "selbständig und individuell arbeiten konnte», indem man "dort länger verweilte und ins Detail ging, wo es einen interessierte».

\section{Fazit}

Im Vergleich mit den anderen, bekannten Unterrichtsmethoden nimmt der Werkstattunterricht bezüglich sozialen Lernens und unterrichtlicher Strukturierung eine Mittelstellung ein. Er ermöglicht Gemeinschaftsbildung, vermag aber auch den Forderungen nach Individualisierung des Lernens nachzukommen. Das breite, vom Lehrer vorstrukturierte Lernangebot verlangt vom Lernenden mehr Verantwortung für sein eigenes Lernen, billigt ihm aber auch eine gewisse Wahl- und Entscheidungsfreiheit zu. Daß die mit Werkstattarbeit angestrebten pädagogisch-didaktischen Ziele tatsächlich auch erreicht werden können, belegen die oben aufgeführten Schüleräußerungen. Das Beispiel der Werkstatt «Klima und Wetter» zeigt, daß sich der Aufwand, der mit Werkstattunterricht verbunden ist, in der Regel für alle Beteiligten lohnt. Es bleibt zu hoffen, daß dieser Bericht auch andere Geographielehrerinnen und -lehrer dazu anregt, mit Lernwerkstätten Erfahrungen zu sammeln und ihren Unterricht dadurch methodisch zu bereichern.

\section{Literatur}

BAUER, J., et al. (1989): Physische Geographie. Mensch und Raum. Cornelsen \& Schroedel, Berlin.

BERIGER, S. (1992): Wasser Werkstatt. Zytglogge Werkbuch. Zytglogge Verlag, Bern.

EDELMANN, W. (1993): Lernpsychologie. Beltz, Psychologische Verlagsunion, Weinheim.

FREI, J., \& HOFFMANN, A. (1992): Dinosaurier. Unterlagen für den individualisierenden und gemeinschaftsbildenden Unterricht. Verlag der ZKM, Zell.

FREY, K., \& FREY-EILING, A. (1992): Allgemeine Didaktik. VdF, Zürich.

FREY, K., \& FREY-EILING, A. (1995): Manual zur Entwicklung von Werkstattunterricht. Publikation des Instituts für Verhaltenswissenschaft, ETH Zürich.

KELLER, H. (1995): Hintergründe nach Erweiterung der Unterrichtsformen. Vortrag an der Praktikumsleitertagung am 9.3.1995 der Universität Zürich, Abteilung Höheres Lehramt. GASSER, P. (1992): Didaktische Impulse, Gerlafingen.

GASSER, P. (1995): Neue Lernkultur - eine integrative Didaktik, Gerlafingen.

LANDOLD, H. (1994): Erfolgreich Lernen und Lehren. Pädagogik bei Sauerländer, Aarau.

LANDWEHR, N. (1994): Neue Wege der Wissensvermittlung. Pädagogik bei Sauerländer, Aarau.

PALLASCH, W., \& REIMERS, H. (1990): Pädagogische Werkstattarbeit. Pädagogisches Training bei Juventa, Weinheim \& München.

REICHEN, J. (1991): Sachunterricht und Sachbegegnung. Sabe Verlag, Zürich.

SLABY, P. (1993): Wir erforschen den Boden. Materialien für den Unterricht. Verlag "Die Werkstatt"/AOL-Verlag, Oberkirch.

WEBER, A. (1991): Erfahrungen im individualisierenden und gemeinschaftsbildenden Unterricht. Verlag der Zürcher Mittelstufenkonferenz, Zell.

SCHWEIZERISCHER BUNDESRAT \& EDK (1995): Verordnung des Bundesrates/Reglement der EDK über die Anerkennung von gymnasialen Maturitätsausweisen (MAR) vom 16. Januar/15. Februar 1995, Bern.

STASCHEIT, W., \& KNEIP, W. (1991): Wasser erforschen und erfahren. Verlag an der Ruhr, Mülheim.

SCHOCH-PERRET, E. (1994): Erfolgreiches Lernen mit dem Werkstatt-Unterricht. Schule aktuell, 18-37. E. Schatz Verlag, St. Gallen.

ZÜRCHER, K. (1987): Werkstatt-Unterricht $1 \times 1$. Didaktisches und Praktisches. Zytglogge Werkbuch. Zytglogge Verlag, Bern. 